\title{
IDENTITE ET RAPPORTS DE FORCE DANS LE CANDOMBE ARGENTIN ${ }^{1}$
}

\author{
- JEAN-ARSĖNE YAO \\ https:/ /orcid.org/0000-0002-5002-5658 \\ Université Félix Houphouët-Boigny (Côte d'Ivoire)
}

\section{RÉSUMÉ}

La domination espagnole en Amérique après sa découverte et sa conquête a conduit, comme on le sait, à l'introduction de l'esclavage noir sur ce continent. En Argentine, l'une des principales empreintes laissées par les esclavagisés africains et leurs descendants, selon l'historiographie, est le Candombe, que ceux-ci revendiquent comme propre à eux. Maintes fois interdite par l'autorité blanche, parce que "obscène", mais surtout pour éviter les atroupements suceptibles de favoriser une revolte, cette danse populaire par excellence a été utilisée pour construire une identité différentielle par rapport aux autres groupes. Et partant, a constitué un cadre de subversion contre la domination blanche. C'est peu dire qu'en tant que lieu de rencontre de la population noire, le Candombe a favorisé une mobilité vers la recherche d'une identité africaine. Le présent travail propose, d'une part, une approximation aux stratégies utilisées par les Afro-Argentins dans le Candombe pour déjouer les tentatives de déshumanisation menées par leurs maîtres. Et d'autre part, d'analyser les représentations actuelles de cette danse dans la société argentine caractérisée par le mythe de la nation blanche sans présence "noire".

Mots clés: Emprunte. Esclavage. Argentine. Musique. Candombe.

\section{RESUMO IDENTIDADE E RELAÇÕES DE FORÇA NO CANDOMBE ARGENTINO}

A dominação espanhol na América após sua descoberta e conquista levou, como sabemos, à introdução da escravidão negra neste continente. Na Argentina, um dos principais vestígios deixados pelos africanos escravizados e seus descendentes, de acordo com a historiografia, é o Candombe, que eles afirmam ser seus. Muitas vezes proibida pela autoridade branca, porque "obscena" , mas principalmente

1 Le présent article est issue d'une recherche menée dans le cadre du projet "La afrodescendencia americana y su contribución al Buen Vivir", coordonnée par le Dr. Teresa Cañedo-Argüelles Fábrega, de l'Université d'Alcalá, et fait partie d'un appel à candidatures conjoint entre cette institution et la Casa de Velázquez, en Espagne. 
para evitar bandos propensos a uma revolta, essa dança popular por excelência foi usada para construir uma identidade diferencial em relação aos outros grupos. E assim, constituiu uma estrutura de subversão contra a dominação branca. Não é dificil dizer que, como local de encontro da população negra, Candombe promoveu a mobilidade em busca da identidade africana. O presente trabalho propõe, por um lado, uma aproximação às estratégias utilizadas pelos afro-argentinos em Candombe para frustrar as tentativas de desumanização realizadas por seus senhores. Por outro lado, analise as representações atuais dessa dança na sociedade argentina caracterizada pelo mito da nação branca sem presença "negra".

Palavras-chave: Impressão. Escravidão. Argentina. Música. Candombe.

IDENTITY AND FORCE RELATIONS IN THE ARGENTINE CANDOMBE

The Spanish domination in America after its discovery and conquest led, as we know, to the introduction of black slavery on this continent. In Argentina, one of the main traces left by the enslaved Africans and their descendants, according to the historiography, is the Candombe, which they claim as own to them. Many times forbidden by the white authority, because "obscene", but especially to avoid the flocks likely to favour a revolt, this popular dance by excellence was used to build a differential identity compared to the other groups. Thus, constituted a framework of subversion against the white domination. It is hardly saying that as a meeting place for the black population, Candombe has promoted mobility towards the search for an African identity. The present article proposes, on the one hand, an approximation to the strategies used by the Afro-Argentineans in Candombe to thwart the attempts of dehumanization led by their masters. On the other hand, to analyse the current representations of this dance in the Argentine society characterized by the myth of the white nation without "black" presence.

Keywords: Heritage. Slavery. Argentina. Music. Candombe.

RESUMEN IDENTIDAD Y RELACIONES DE PODER EN EL

\section{CANDOMBE ARGENTINO}

La dominación española en América después de su descubrimiento y conquista llevó, como es sabido, a la introducción de la esclavitud de los negros en este continente. En Argentina, una de las principales huellas dejadas por los esclavos africanos y sus descendientes, según la historiografia, es el Candombe, que estos reivindican como propio. 
A menudo prohibida por las autoridades blancas por ser «obscena», pero sobre todo para evitar las reuniones que podrían favorecer una revuelta, esta danza popular por excelencia ha servido para construir una identidad diferenciada con los demás grupos. Por ende, constituyó un marco de subversión contra la dominación blanca. Es un eufemismo decir que, como lugar de encuentro de la población negra, el Candombe fomentaba la movilidad en la búsqueda de una identidad africana. El presente trabajo propone, por una parte, una aproximación a las estrategias utilizadas por los afroargentinos en el Candombe para frustrar los intentos de deshumanización llevados a cabo por sus amos. Y, por otro lado, analizar las representaciones actuales de esta danza en la sociedad argentina caracterizada por el mito de la nación blanca sin presencia «negra».

Palabras clave: Herencia. Esclavitud. Argentina. Música. Candombe.

\section{Introduction}

La contribution africaine - issue principalement de l'esclavage - à la configuration de l'identité historique et culturelle de l'Argentine a un poids et un sens encore peu connus et valorisés dans la conscience commune de la population. Ce fait est la conséquence de la dissimulation phénotypique et culturelle des noirs qui a comme corollaire la négation de leur passé, leur présence actuelle et leurs droits. Cette exclusion a contribué à l'invisibilité de leur rôle auprès des Indiens et des Européens dans l'édification de la nation. D'où l'idée d'une Argentine sans Noirs, expression d'une construction idéologique faisant du stéréotype «nation de race blanche et de culture européenne" un élément clé de l'identité argentine» (CIRIO, 2009, p. 17).

En dépit de cela, il faut dire que dans la recherche de moyens de maintenir des liens avec l'Afrique, les esclavagisés dans ce pays, de même que ceux des autres régions de l'Amérique, ont utilisé la culture comme mécanisme pour l'affirmation de leur propre identité. C'est d'ailleurs à juste titre que Ngou-Mve déclare que:
[...] lo que se ha introducido en este continente -América- desde el siglo XV hasta el XIX, no fueron toneladas de ébano, sino hombres y mujeres cargados de cultura, de una cultura africana milenaria, que ellos no podían haber olvidado por el simple hecho de cruzar el Atlántico en condiciones inhumanas. (2001, p. 61)

En Argentine, l'une des principales empreintes laissées par les esclavagisés africains et leurs descendants, selon l'historiographie, est le Candombe, que ceux-ci revendiquent comme propre à eux. Maintes fois interdite par l'autorité blanche, parce que "obscène", mais surtout pour éviter les atroupements suceptibles de favoriser une revolte, cette danse populaire par excellence a été utilisée pour construire une identité différentielle par rapport aux autres groupes. Et partant, a constitué un cadre de subversion contre la domination blanche. C'est peu dire qu'en tant que lieu de rencontre de la population noire, le Candombe a favorisé une mobilité vers la recherche d'une identité africaine.

Mais parler d'identité culturelle, c'est aussi parler de rapports de forces, redéploiement 
idéologique et législations d'exception. En ce sens, je considère d'une grande importance les espaces qui peuvent être gagnés dans cette lutte par les afro-descendants, car, d'une manière ou d'une autre, ils re-configurent les dispositions du pouvoir, faisant pencher la balance d'un côté ou de l'autre. Il s'agit ici aussi de parler du processus de racialisation à l'ère multiculturelle dans le contexte spécifique de l'Argentine actuelle. La racialisation étant prise comme la démarche de construction des rapports de domination à travers la détermination des différences humaines - culturelles ou phénotypiques - et des relations sociales. En définitve, c'est un processus dialectique, politique, social, culturel et cognitif de construction des différences qui servent de base à la hiérarchisation des groupes humains (HELLEBRANDOVA, 2014).

Comment et dans quelle mesure le Candombe a été utilisé pour remettre en cause les rapports sociaux et de pouvoir à partir de l'expérience vécue par les Argentins d'origine africaine ? Pour répondre à cette question, mon hypothèse est que les Afro-argentins ont constamment utilisé ce rythme dans la production de représentations de leur culture en tant qu'ensemble de symboles de leur vie. Je m'appuie aussi sur la perspective afro-diasporique développée par Lao-Montes qui vise à «repenser l'être, la mémoire, la culture et le pouvoir au-delà des frontières de la nation en tant qu'unité d'analyse» (2007, p. 309). Cela implique de promouvoir fondamentalement une vision alternative à celle offerte par le discours hégémonique.

La stratégie méthodologique globale s'est basée sur l'examen des discours publics prononcés dans divers domaines et événements rassemblant les candomberos. Par ailleurs, les données analysées dans ce travail ont été construites à partir des pratiques qui configurent le Candombe comme un élément de subversion du point de vue de ses protagonistes.
D'autre part, il convient de noter qu'outre la littérature spécifique sur le Candombe (GELER, 2011; GHIDOLI, 2010; FRIGERIO et LAMBORGHINI, 2009; CIRIO, 2007; LÓPEZ, 1999; VILLANUEVA, 1980), la presse afro-argentine et les documents produits par les collectifs candomberos - déclarations imprimées ou sur Internet - ont également contribué au corpus.

Inscrit dans le débat anthropologique sur les identités, qui habituellement tourne autour de l'essentialisme et du constructivisme, mon étude vise à réfléchir sur le Candombe en tant qu'instance d'expression/représentation, de protestation et de résistance culturelle. La base conceptuelle de ma réflexion c'est la notion de culture comme recours de Yúdice (2002), qui permet une approche à la manière dont certaines demandes sociales utilisent la musique pour être atteintes. Il s'agit donc d'une réflexion sur l'un des héritages laissés par l'esclavage dans la société argentine et les vicissitudes de sa permanence, à partir d'un «appareil conceptuel éloigné des hypothèses universelles euro-occidentales dans l'analyse culturelle» (FERREIRA, 2008, p. 92).

Pour ce faire, mon propos s'articulera en quatre temps. Tout d'abord, j'analyserai l'influence de l'imaginaire colonial sur la narrative hegémonique de la nation argentine, puisque la colonialité du pouvoir a soutenu la colonialité du savoir. Puis je me pencherai sur le Candombé comme contestation de la racialisation des Afro-argentins, pour ensuite analyser ses avatares dans la construction de l'identité argentine. Et finalement je mettrai en relief le rôle joué par cette expression culturelle dans la reconquête de nouveaux espaces sociaux.

\section{Le Candombe face à l'imaginaire colonial}

Dans l'abondante littérature sur le Candombe, il existe une certaine unanimité sur le fait que 
ce vocable désigne à la fois une musique, une danse et une fête, dont les premières références connues datent du XVIIlème siècle. Il s'agit de mesures d'interdiction émises par les autorités contre sa pratique. En effet, dans un esprit qui ne favorisait pas l'exécution de danses "exotiques", le Gouverneur du Río de la Plata, Juan José de Vértiz, a promulgué un édit le 20 septembre 1770:

Que se prohiben los bayles indecentes que al toque de su tambor acostumbran los negros, si bien podrán públicamente bailar aquellas danzas de que usan en las fiestas que se celebran en esta ciudad [...] todo vajo la pena de doscientos azotes, y de un mes de barranca a los que contraviniesen. (MATA LINARES, p. 192-198)

Même si le substantif Candombe n'apparait pas dans cet arrêté, la référence au tambour, instrument principal de ce rythme, montre bien sa pratique. Généralement dans les locaux servant de sièges aux nations africaines², sortes d'associations créées par les colons espagnols dont l'objectif principal était d'empêcher la germination d'une conscience collective de peuple opprimé chez les esclavagisés. Véritable réfuge d'africanité, «El barrio donde dominaba la población africana se llamaba barrio del Tambor, porque era el instrumento favorito de sus candombes, música monótona y bailes enteramente africanos» (QUESADA, 1998, p. 87).

Également appelés "barrios del mondogo", ces colonies d'esclaves noirs ont toujours suscité la suspicion des autorités coloniales. En fait, les nations n'ont jamais marché comme les autorités s'y attendaient. En effet, bien que leur fonctionnement externe semblait totalement contrôlé par le pouvoir colonial, en revanche, leur dynamique interne permettait de renforcer l'ethnicité (SCHÁVELZON, 2003). Pour preuve, la déposition d'un officier du Cabildo

2 Dans d'autres pays des Amériques, ces groupes ont été appelés "Cabildos". selon laquelle “Un grupo grande se juntaban a la noche en número de trescientos para danzar indecentemente" (CHAMOSA, 2003, p. 357).

En agissant de la sorte, les noirs esclavagisés entendaient la culture comme un mode de vie, plutôt qu'un simple ensemble d'expressions esthétiques et symboliques (WILLIAMS, 1988, p. 91). Mieux, ce refus d'obtempérer était une forme de lutte pour leurs conditions matérielles (ESCOBAR, 1992, p. 69). Et contre l'impérialisme culturel basé sur le pouvoir d'universaliser les valeurs de la classe dominante en faisant méconnaitre celles des minorités (BOURDIEU et WACQUANT, 1998, p. 109). En se cachant pour le danser, les Afro-Argentins revendiquaient le Candombe comme un des éléments avec lequel ils devaient construire leur identité différentielle par rapport aux autres groupes.

Il ne fait aucun doute que la décision du Gouverneur Francisco de Paula Bucarely, le 3 novembre 1776, selon laquelle "Que no se permitan los bayles indecentes que acostumbran tener los negros" (MATA LINARES, folios 179-185) était aussi une manière d'affronter la multiculturalité avec la vision de ceux pour qui la société générique passe par l'abolition de la différence. $\mathrm{Ce}$, dans la poursuite de la construction d'une colonie dont les traits dominants seraient essentiellement ceux de la civilisation occidentale. Contre cette démarche, les sièges des nations, contrôlées plus directement par les Africains eux-mêmes, ont été utilisées par ceux-ci pour défendre leurs intérêts avec une organisation de type monarchique, avec "rois" et "reines", ce que les autorités blanches voyaient comme une insubordination, voire un affront. Puisqu'en faisant allégence à leurs "monarques", les Afro-Argentins recréaient leur société africaines où ceux-ci étaient les garants de la tradition.

C'est justement pour ça qu'en 1788, le procureur général demanda l'interdiction de ces danses pour les raisons suivantes: 
Se originan en ellos una manifesta ruina de las almas con las muchas y graves ofensas, que hazen a Dios, porque otra cosa son estos bayles, sino unos verdaderos Lupanares donde la concupiscencia tiene el principal lugar haze todo lo agradable de ellos con los indecentes, y obscenos movimientos que se executan sin que de otro modo los puedan hazer, pues para ello contribuye el mismo son de sus instrumentos que es el mayor alicitivo para alterar el espiritu haciendo concupiscible, y poniendo en movimiento y disposición de practicar las mismas obscenidades. (KORDÓN, 1968, p. 10)

Ces lieux communs sur la population noire et le Candombe que le colonisateur blanc transforme en marqueur mettent en relief la réalité controversée d'une société qui renvoie aux séquelles de l'exclusion des Noirs. Toutefois, comme il est public et notoire, ce processus coercitif n'a pas atteint ses objectifs, puisque la résistance culturelle noire configurée au cours des générations coloniales a permis la reproduction de sociétés et de cultures dans des conditions où leur survie était presque impossible. L'espace colonial devient, comme le suggère Pratt, une zone de contact, "un espacio social en el que culturas desiguales se encuentran, chocan y luchan entre sí, a menudo, en relaciones altamente asimétricas de dominio y subordinación como el colonialismo, la esclavitud" (2010, p. 4).

Contre la construction d'une culture arbitrairement homogénéisée, les noires ont recherché la reconnaissance de l'exercice de la différence dans une société culturellement différenciées. Plus qu'une simple "tolérance", telle que souligner par Taylor (1993), ils aspiraient au plein exercice de leur culture y compris le droit à un cadre spatial à sa reproduction. Les témoignages de l'époque donnaient toujours une image adverse des rythmes et danses afro-argentins. Il suffit de lire cette chronique du voyageur Concolorcorvo qui a décrit la danse des noirs de la manière suivante :
Las diversiones de los negros bozales son las más bárbaras y groseras que se puedan imaginar: su canto es un aúllo. De ver sólo los instrumentos de su música se inferirá lo desagradable de su sonido. La quijada de un asno con su dentadura floja, con las cuerdas de su principal instrumento que rascan con un hueso de carnero, asta u otro palo duro [...], hacen unos altos y tiples tan fastidiosos y desagradables que provocan a tapar los oídos o a correr a los burros, que son los animales más estólidos y menos espantadizos. En lugar del agradable tamborcillo de los indios, usan los africanos un tronco hueco y a los dos extremos le ciñen un pellejo tosco golpeando el cuero con sus puntas, sin orden, sólo con el de hacer ruido. Sus danzas se reducen a menear la barriga y las caderas con mucha deshonestidad, a las que acompañan con gestos ridículos y que traen a la imaginación la fiesta que hacen al diablo los brujos en sus sábados, y finalmente sólo se parecen las diversiones de los negros a las de los indios, en que todos brincan y finalizan, en borracheras. (CONCOLORCORVO, 1942, p. 159)

Ce témoignage qui démontre toute l'ignorance de son auteur de la culture africaine renforce les clichés sur le Candombe, puisque "La representación tiene por función perpetuar y justificar la diferenciación social, puede como los estereotipos - pretender la discriminación o mantener una distancia social entre los grupos respectivos" (ABRIC, 2001). Toutefois, il permet au moins d'apprécier son développement. D'autre part, avec leur présence active dans la sphère publique et dans la vie quotidienne, les noirs défient les autorités et élargissent la définition de leur communauté. Ils montrent par ailleurs dans quelle mesure ils ne sont pas seulement une composante de la population argentine, avec leur contribution à la construction de la société national, mais également qu'ils sont sujets à la discrimination raciale.

Cependant, malgré les interdictions et le rejet de la société coloniale, les Candombes 
étaient parfois autorisés ${ }^{3}$. Ces autorisations ont coïncidé avec les moments de plus grande splendeur du Candombe qui se déroulait dans les maisons, les voies publiques, les veillées et les sièges des nations africaines. Les témoignages abondent au sujet de l'intervention de foules africaines dans les célébrations à l'époque de Juan Manuel de Rosas, alors Gouverneur de Buenos Aires. Les Noirs venaient jouer leurs Candombes même sur la Plaza de la Victoria, l'actuelle Plaza de Mayo (CONCOLORCOVO, 1942). Mais après la seconde moitié du XIXe siècle, les auteurs soulignent l'extinction significative de ces danses (ANDREWS, 1989, p. 187-203), du moins dans leur forme publique. Cela a amené chroniqueurs, chercheurs et historiens à annoncer la "disparition" du Candombe. Ortiz Oderigo a déclaré ce qui suit:

La vivencia del candombe, como música, como danza y como ceremonia folklóricas, se mantuvo hasta la caída de Rosas. Mermó su intensidad al disminuir el elemento afroargentina y cuando el alud inmigratorio "blanqueo" la heterogenia textura etnográfica de nuestro país. [...] Afines del siglo pasado, sólo afloraba durante las fiestas del carnaval [...] Podemos fijar el decenio de 1870 como la época en que comienza [...] su inevitable decadencia. (1980, p. 77)

Les Afro-argentins ont été victimes de la construction de l'état uninational sur un espace multiethnique et multiculturel; diversité qui a été historiquement perçue comme un obstacle à la construction d'États "modernes", avec une communauté culturellement homogène. En ce sens, la différence constitue un affront au modèle étatique où le rachat de l'altérité passe par l'assimilation à un modèle référentiel plus imaginaire qu'objectif.

3 AGN, X - 31 - 11 - 5. En 1795, la "Nation Congo" fut autorisée à exécuter ses danses le dimanche et les jours fériés. En 1799, ce fut le tour de la "Nation Cambunda".

\section{Le Candombe comme subversion}

\section{carnavalesque}

Fondamentalement populaire, le carnaval est une fête de masse et musicale qui recherche le plaisir et le divertissement, ainsi que la suppression des limites qui empêchent le plein épanouissement des individus dans la vie quotidienne. C'est dire que dans cette célébration, les valeurs et les normes sociales, les comportements établis et les modèles esthétiques sont modifiés et recréés dans un nouvel univers d'ordres symboliques qui permettent l'émergence de groupes de "rebelles" dans un système définit. Dans cet espace, les cultures populaires sont affirmées et revitalisées pour faire face aux cultures dominantes. En Argentine, les Noirs ont formé des sociétés philharmoniques appelées comparsas avec des noms assez évocateurs : "Los Negros Santafesinos" (PISTONE, 1996, p. 10), "Progreso del Plata", “Estrella del Sud", “Los Negros Mozambique", “La Lira Argentina", "Flor de Cuba", "Negros Unidos Argentinos", "Los Negros Candomberos", “Los Parias Entrerrianos" (VILLANUEVA, 1980, p. 56).

De cette façon, la culture noire connaît également un processus d'objectivation dans la mesure où il apparaît comme objet d'un certains activisme noirs qui revendique la contribution de ce collectif à la vie du pays et luttent contre sa marginalisation. Les Afro-argentins ont donc eu recours à des éléments de la sphère publique et définis par des formes hégémoniques, afin de mieux contrôler leur existence. En période de carnaval, ces troupes exécutaient le Candombe qui n'était pas du goût de la société la plus distinguée qui s'en est toujours plaint, même si cette musique avait fini par rythmer leur vie, comme le dit si bien Estrada:

¿Quién no ha sentido alguna vez el ruido del rustico instrumento, a cuyo compás danzan los negros en el tambor? ¿Quién no ha tenido la cu- 
riosidad de ver sus diversiones u oído hablar a sus mayores de un día en que la negra vieja de la casa era engalanada con ricos atavíos para ser la Reina de la fiesta? (1863, p. 15)

Le sens représentatif de cette musique, sa "rébellion" et ses relations avec les blancs, lui était inséparable de l'expérience d'écoute et de danse. C'est aussi un bon exemple d'une forme d'engagement à la fois matérielle et symbolique, un processus dans lequel le Candombe représente un projet social d'ordre collectif. Clairement, les comparsas permettaient de construire une identité culturelle en tant que réalité vécue, une manière d'être et de vivre leur époque.

Cette perception romantique du Candombe ne cache pas moins les tensions entre l'oligarchie blanche et la communauté noire dont la musique représentait pour les intellectuels le summum de la barbarie et l'antithèse de la "civilisation et de la modernité" recherchés par la société argentine. Le description faite par Ramos Mejía est assez éloquente:

[...] Los candombes empezaban a fermentar con la alegría gritona y agitante de los negros en libertad [...] Porque la fauna séptica se insinuaba en el alma de todos, despertando aquellos apetitos que el voluptuoso presentimiento del manoseo de las niñas y señoras movilizaba de un modo brutal [...] (1907, p. 334-336)

L'auteur souligne la charge sexualisée qui érodait les limites de la moralité et le métissage entre les classes sociales qu'avait, selon lui, les Candombes de manière prédominante. Précisément, à ces danses assistaient Rosas, ce qui fut décrits et réprimés par l'intelligentsia locale, comme un signe du peu de penchant du gouverneur pour la civilisation et de son intention de manipuler les masses. Il n'est donc pas surprenant que, dans l'une des rares images représentant ces danses, prise par les chercheurs et les écrivains du XXe siècle comme une sorte de "reflejo naturalista de la realidad" (GHIDOLI, 2010), Martín Boneo a capturé un candombe en présence de Rosas et famille.

Otage des conflits politiques entre Unitaires et Fédéraux ${ }^{4}$, le Candombe a été embastillé dans une histoire de censure et autorisation, de réprobation et acceptation attachée à un imaginaire marginal. Et comme le dit à juste titre Lavou Zoungbo (2011), ce schéma de représentation du Noir pourrait s'expliquer par l'existence d'une création discursive et, surtout, d'une formation idéologique héritée des pays européens impliqués dans les aventures coloniales (2011, p. 67).

Et comme les lignes qui précèdent l'ont démontré, dans la description du Candombe l'on mettait en relief, d'un point de vue blanc-occidental, les mouvements de danse sexués, en désaccord avec la forte proscription corporelle et idéologique imposés aux femmes et aux hommes bourgeois blancs (BARRANCOS, 2008). Et au son de leurs tambours - qui étaient considérés comme infernaux -, associant les Candombes à la sauvagerie et à la débauche d'une époque également considérée comme barbare.

[Los negros] se dieron organización según sus hábitos y Reyes según los usos y jerarquías que probablemente traían desde sus tierras africanas. Los domingos y días de fiesta, ejecutaban sus bailes salvajes, hombres y mujeres a la ron$\mathrm{da}$, cantando sus refranes en sus propias lenguas al compás de tamboriles y bombos grotescos. La salvaje algazara que se levantaba al aire, de aquella circunvalación exterior, la oíamos (hablo como testigo) como un rumor siniestro y ominoso desde las calles del centro, semejante al de una amenazante invasión de tribus africanas, negras y desnudas. Desde que subió al gobierno, Rosas se hizo asistente asiduo de los Tambos. (LÓPEZ, 1896, p. 365-366)

4 Les Unitaires défendaient la création d'un État avec un pouvoir centralisé à Buenos Aires, contrirement aux Fédéraux qui optaient pour une fédération de provinces autonomes. 
Ces images ont progressivement gagné en complexité lorsque les caractérisations raciales étaient combinées à des variables telles que le sexe et la promiscuité. Ces descriptions reposaient sur la pertinence donnée, dans une perspective eurocentrique, aux mouvements de danse sexués qui étaient en dehors du canon imposé à la population blanche (GELER, 2011). De telles manifestations étaient preçues comme inesthétiques, déplaisantes et gênantes, presque comme un vice.

La crainte du Noir qui se dégage de ces descriptions était basée sur les préjugés à leur égard. À la suite de ces représentations, il a été considéré - aujourd'hui encore - que la majorité de la population d'ascendance africaine pourrait difficilement manifester un comportement "civilisé". Mais toutes ces choses étaient permises pendant les carnavals comme l'émergence dans les corps de "impulso orgiástico reprimido" (BAJTíN, 2005, p. 225). La description de Ramos Mejía d'un de ces Candombes se focalisait sur la barbarie - "pintarrajeados tambores tocaban un ruido del más desastroso efecto" - et sur les corps en mouvement:

Sudorosos y fatigados por la larga peregrinación marchaban sin embargo con cierto desembarazo vertiginoso, imprimiendo al cuerpo movimientos de una lascivia solemne y grotesca. Las negras, muchas de ellas jóvenes y esbeltas, luciendo las desnudeces de sus carnes bien nutridas, revelaban en sus rostros alegres, un ánimo satisfecho y despreocupado. Las gráciles Venus imponían con indolencia las mamas rotundas como una expresión de su poder fecundante. (RAMOS, 1907, p. 329-330)

L'érotisme de la scène est évident, approfondissant les idées qui exacerbent l'association entre noirceur et hypersexualité au détriment de la chasteté, considérée comme une valeur idéale des sociétés civilisées (c'està-dire blanches). Encore une fois, je voudrais remarquer le grand fardeau de fantaisie et de désir qui est révélé dans les mots de l'auteur. Ces portraits s'inscrivent dans le processus en cours qui a vu le jour depuis l'origine de l'esclavage de stéréotypes sexuels / raciaux dans l'Atlantique, qui marquent encore les femmes et les hommes noirs.

Et comme dans tout stéréotype colonial, selon Bhabha (20202), "l'Autre" est construit simultanément comme objet de désir et de dérision, possible dans l'articulation qui inscrit le corps colonial dans des relations de plaisir et de désir, et dans ceux de domination et de pouvoir, en établissant un "rango de diferencias y discriminaciones que conforman las prácticas discursivas y políticas de la jerarquización racial y cultural" (2002, p. 92).

D'où le désir des Noirs de démonter ces descriptions qui ont naturalisé leurs différences et qui ont entraîné l'impulsion de l'endoracisme par laquelle beaucoup d'entre eux ont fini par présumer que leur comportement était porteur de barbarie dont l'éloignement était nécessaire pour s'intégrer à la nation moderne et civilisée.

\section{Entre être et paraître}

Au XIXe et au début du XXe siècle, le besoin de créer un "nous" collectif a conduit les dirigeants argentins à diffuser des modèles culturels, des mythes d'origine et un ensemble de symboles visant à consolider l'identité argentine. Les héros de la nationalité argentine - Bartolomé Mitre, Juan Bautista Alberdi, Esteban Echeverría, Domingo Faustino Sarmiento et bien d'autres - ont reproduit des vertus éthiques qu'ils ont projeté sur l'imaginaire collectif comme une sorte de miroir à partir duquel devaient se forger les vertus nationales. Ils ont promu l'immigration européenne qui selon eux devait permettre de façoner des citoyens "blanchis" en couleur et "européanisés" dans les mentalités et les us. 
Alberdi (1852), soutenait que les Argentins étaient des Européens adaptés pour vivre en Amérique. En outre, il a déclaré: "En América todo lo que no es europeo, es bárbaro; no hay más división que ésta: primero el indígena, es decir el salvaje; segundo, el europeo, es decir nosotros" (1852, p. 83). Pour sa part, Ingenieros (1913), sans doute le sociologue argentin le plus influent s'appuyant sur le darwinisme social, a déclaré:

La europeización no es en nuestro concepto, un deseo...; es un hecho inevitable, que se produciría aunque todos los hispanoamericanos quisieran impedirlo. Nace de causas determinantes que ya existen ajenas a nuestro deseo. Por una ley sociológica inevitable los agregados sociales más evolucionados se sobreponen a los menos evolucionados... Nos europeizaremos oportunamente, como lo preveía Sarmiento la sociología puede afirmar esa futura transformación de la América Latina. (1913, p. 228-229)

Par conséquent, le passage du noir au mulâtre était considéré comme une amélioration introduite au sein de la population argentine en général et chez les afro-argentins en particulier. Ainsi, dans les années 1860, José Manuel Estrada déclarait qu'il n'y avait presque plus de Noirs à Buenos Aires. En précisant qu'il ne faisait pas référence aux mulâtres, mais aux personnes d'ascendance africaine pure, Estrada soutenait que le mélange de races avait entraîné une amélioration progressive de la population noire et la disparition du véritable type de race éthiopienne (RODRIGUEZ, 1961, p. 110).

Précisément Sarmiento avait publié que: “En la ciudad de Córdoba, ya no quedaban negros. Y muchos de los antiguos mulatos habían pasado a ser caballeros, siguiendo el natural desenvolvimiento y progreso de los tiempos" (1900, p. 71). La minimisation de la diaspora africaine était l'un des mythes fondateurs de la nation argentine créée en fonction des in- térêts des secteurs hégémoniques et qui a été utilisé comme un discours de domination tendant à neutraliser les particularités ethniques. Je dirais même que l'identité nationale s'est forgée aux dépens des identités ethnique et basée sur un projet d'homogénéisation culturelle supposé intégratif. Et cela a eu des effets sur les pratiques musicales des Afro-argentins.

En effet, il existe de nombreuses références qui indiquent l'existence de deux types de groupes artistiques afro-argentins à cette époque-là: les candomberas, fondées dans le sud de Buenos Aires, et les musicaux, situés dans le nord, ce qui indiquait que la situation sociale de leurs membres était un peu meilleure (FRIGERIO et LAMBORGHINI, 2011, p. 21). Alors que les premiers privilégiaient l'héritage culturel africain, les derniers promouvaient une esthétique plus européanisée, privilégiée par les intellectuels noirs, sans doute influencés par l'idéologie des auteurs des fondements de la nation argentine moderne. De ce groupe, plusieurs artistes et compositeurs noirs se sont distingués par leurs compétences dans la musique académique européenne. Parmi ceux-ci, il y avait Zenón Rolón, musicien, compositeur et chef d'orchestre, qui a vécu plusieurs années à Florence; et Manuel Posadas, un violoniste qui a étudié pendant trois ans au Conservatoire de Bruxelles.

Pour cette élite noire et métisse, la recherche de l'égalité avec les Blancs était un objectif recherché depuis longtemps, qui consistait à noyer les souvenir de l'ère candombera de Rosas dans le calice de l'oubli. C'est ce que l'on note dans cet article sur les choses que certains Afro-argentins considéraient comme bonnes et nécessaires pour leur bien-être en tant qu'individu et en tant que groupe social.

Muchas han sido las personas que se han felicitado y nos han felicitado al ver que hemos iniciado la propaganda de modificar u olvidar la costumbre de desempeñar un papel que no 
nos corresponde en los días de carnaval y particularmente a nuestras comparsas, donde existe tanto joven hábil para aprender un instrumento musical. [...] se encuentran personas que pertenecen a varias de esas sociedades y que nos han de ayudar a llevar a cabo esta indispensable innovación ${ }^{5}$.

Il est ici claire que sous l'influence de l'imaginaire dominant, le collectif afro-argentin a fini par intérioriser les préjugés qui le stigmatisent. De ce qui précède, on peut en déduire que, pour être acceptés par les "autres", les Afro-Argentins ont ressenti le besoin d'éviter l'image négative que l'on prêtait aux arts d'origine africaine. L'un des effets de cette vision est que, apprendre à jouer des instruments de musique d'origine européenne était devenu un emblème de la modernité et du progrès, ce qui a permis de démontrer "la diferencia que hay entre una sociedad candombera que causa la hilaridad de todos y una sociedad musical que recibe, siempre, el aplauso general"6.

Ainsi se révèle le mépris vis-à-vis du Candombe dans la sphère intime de la communauté noire renforcé par l'idéal du blanchiment en tant que pratiques de l'oppression qui articules les relations sociales et de pouvoir racialisées. Mais qui sont invisibles et naturalisés à travers une série de pratiques, d'expressions ou d'images qui marquent la vie de tous les jours. Ce rejet du Candombe montre aussi la formation d'une vision racialisée développée sous l'idéologie du métissage, qui impose l'idéal blanc comme le seul modèle auquel l'on puisse aspirer.

L'on trouve ici un stéréotype de la culture noire basé sur des représentations plus ou moins générales de l'acceptation sociale. Ce stéréotype a du sens en faisant référence à sa composante individuelle et à ses coordonnées sociales. Le journal La Juventud le marque clairement en déclarant: "En estos momentos

5 La Broma, 9 de marzo de 1882.

6 La Broma, 3 de marzo de 1882. trabajamos activamente en el sentido de fundar un gran centro social sobre la base sólida de la unión, y cuyo objetivo principal será el de difundir la ilustración en el seno de nuestra comunidad"7.

Une approche déconstructive permet de se rendre compte que la stigmatisation qui a historiquement pesé sur le Candombe en tant que pratique marginalisée a conduit à l'élaboration de stratégies personnelles comment s'éloigner des tambour (FERREIRA, 2003).

\section{Le Candombe reconquête de}

\section{nouveaux espaces}

Alors que beaucoup parlaient de la "mort" du Candombe, Narciso Binayán Carmona affirmait que dans les années 1940,

Los afroargentinos bailaban en el Shimmy Club desde la medianoche hasta el amanecer bailes afroamericanos: candombe, rumba abierta y una mezcla de ambos. Hubo y debe seguir una viva discusión entre los tradicionalistas y los modernistas, y en algunos momentos hubo dos grupos rivales de tambores y danzarines. (1980, p. 72)

De cette manière, nous avons un concept d'identité stratégique et de position construit de différentes manières "a través de discursos, prácticas y posiciones diferentes, a menudo cruzados y antagónicos [...] sujetas a una historización radical, y en un constante proceso de cambio y transformación" (HALL, 2003, p. 17). D'ailleurs, ce même auteur signale que:

Dentro de la cultura, la marginalidad, si bien permanece en la periferia de la amplia tendencia cultural, nunca ha sido un espacio tan productivo como lo es ahora. $Y$ esto no representa simplemente una apertura por la cual aquellos que están afuera pueden ocupar los espacios dominantes. Es también el resultado de la política cultural de la diferencia, de las luchas sobre la diferencia, de la producción de nuevas

$7 \quad$ La Juventud, 10 de enero de 1879. 
identidades, de la aparición de nuevos sujetos en el escenario político y cultural. (HALL, 2010, p. 289)

Eh bien, la stigmatisation et le trinôme contrôle-réglementation-répression du Candombe et du «noir» dans l'espace public, caractéristiques du contexte argentin du XIXe siècle ont continué au XXe siècle. En effet, après ce que je qualifirais de létargie, le Candombe retrouve de la voix dans les années 1980 à la faveur du retour à la démocratie en Argentine. Toutefois, dans ce contexte qui devait leur être favorable, les pratiquants du Candombe ont rencontré de multiples difficultés et formes de résistance sociale à leur présence. Les appels au rassemblement, connus comme "Llamadas de tambores" ont été, dès le début, fréquemment entravés et/ou surveillée par la Police, tout comme se multiplièrent les plaintes des populations reveraines des lieux de regroupement pour "bruits gênants".

Dans la ville de Buenos Aires, par exemple, les conflits des candomberos avec différents agents et acteurs sociaux se sont exacerbés par l'absence de législation en faveur de la pratique du Candombe dans sa spécificité. Ces démêlés était essentiellement liés à la persistance de l'image de ville blanche, européenne et moderne, où seule la culture d'élite a le droit d'expression dans l'espace public. Et comme le soutiennent Frigerio et Lamborghini:

Más allá de los discursos que puedan articular los candomberos proponiendo imaginarios urbanos, nuevas formas de leer el espacio, la cultura y la historia de la ciudad -que no son igualmente explicitados por todos los grupos-, el sólo hecho de la presencia y el sonido de varios tambores en el espacio público ya constituye una amenaza al orden racial-espacial que sustenta dicha imagen. $(2009$, p. 107)

La citation précédente revèle le caractère politique de la culture dont "los significados son constitutivos de procesos, que implícita o explícitamente, buscan redefinir el poder social" (ÁLVAREZ, 1998, p. 7). En ce sens, les candomberos mettent en œuvre des stratégies de légitimation visant à donner une continuité à une tradition artistique de secteurs dominés (CHAVES, 2010). Dans son analyse des actions des candomberos dans l'espace public de la ville de La Plata, dont l'appropriation est souvent conflictuelle, Mariana Chaves met en relief les liens entre "protestation" et "divertissement" (2010, p. 168).

Cette perception de la culture comme un champ de bataille constant est d'ailleurs certifiée par Stuart Hall lorsqu'il propose l'idée d'une «lutte culturelle» jamais terminée, dans laquelle les victoires ou les défaites sont toujours incomplètes et non définitives. C'est de toute évidence le cas du Mouvement afro-culturel qui en 2007 a reçu l'ordre d'abandonner "son" siège, une usine abandonnée et occupée de manière informelle, situé au numéro 313 de la rue Herrera, dans le quartier de Barracas, quatre ans auparavant. En réaction à cette décesion judiciaire, ce collectif s'est mobilisé jusqu'à être reconnus comme groupe d'intérêt social et culturel. Ne s'arrêtant pas en si bon chemin, ils ont esté en justice la Mairie de Buenos Aires pour racisme institutionnel, obtenant en 2009, le cession d'un bâtiment qui abritait jusque-là un centre culturel dans le quartier de Montserrat.

Il ne fait aucun doute que, ainsi, le Candombe est devenu le réceptacle de l'identité des afro-argentins qui la composent et la jouent. Et l'agir des candomberos est vue comme

[...] ciertas formas de acción colectiva diferentes de aquellas basadas en el conflicto histórico que se ubicaba en el Estado y en las divisiones entre clases sociales. En la escena local contemporánea, se trata de organizaciones sociales que emergieron como forma de lucha contra las políticas neoliberales. (VÁZQUEZ, 2008, p. 166) 
En définitve, la notion résistance des candomberos n'est rien d'autre que la manifestation d'un secteur social en position subalternité dans un contexte multiculturel qu'il tente de modifier. Cette résistance est produite à la fois par ceux qui exercent l'action que par ceux qui, par leur position hégémonique, en sont les destinataires: "Supone la presencia de un conflicto y de un objeto social en disputa que convoca a los sectores juveniles en el espacio público. Es de carácter táctico y puede implicar la alianza de diversos colectivos o grupos" (REGUILLO, 2012, p. 43).

\section{Conclusion}

Tout au long de ce travail, je me suis efforcé de montrer que le Candombe a connu une évolution marquée par des conflits, favorisés d'une part par la colonialité du pouvoir, la construction idéologique de l'Agentine; et d'autre part par les opportunités offertes par le contexte multiculturaliste du pays. Comme telle, cette manifestation culturelle de matrice africaine contient l'histoire de la socialisation et des divertissements de la communauté des esclavagisés africains et leurs descendants, de l'époque coloniale à l'ère actuelle.

La majorité blanche a toujours considéré cet élément identitaire et culturel des noirs comme "sauvage", mettant celui-ci est en conflit avec la vision de la culture en tant que forme de vie. D'où le fait que, bien qu'ayant des objectifs très différents, la minorité noire a par moment eu tendance à penser ses activités sous l'angle hégémonique blanc. Ainsi, le Candombe s'est adapté au contexte socio-culturel du moment, en dialogue avec les structures du pouvoir et faisant l'objet de négociations, interdictions et stigmatisations.

Malgré ces premiers résultats obtenus, il me semble que cette étude ne peut se dispenser d'un travail ethnographique de terrain dans lequel je mettrai en œuvre des techniques de nature non directive telles que l'observation, les conversations et les entretiens avec les protagonistes du Candombe.

\section{Bibliographie}

ABRIC, Jean-Claude. Prácticas sociales y representaciones. México D.F: Ediciones Coyoacán, 2001.

ALBERDI, Juan Bautista. Bases y puntos de partida para la organización política de la República Argentina. Buenos Aires: Museo Mitre, 1852.

ÁLVAREZ, Sonia; DAGNINO, Evelina; ESCOBAR, Arturo. Cultures of politics, politics of cultures: re-visioning Latina American Social movements. Boulder: Westview Press, 1998.

ANDREWS, George Reid. Los afroargentinos de Buenos Aires. Buenos Aires: Ediciones de la Flor, 1989.

BAJTíN, Mijail. La cultura popular en la Edad Media y en el Renacimiento. El contexto de François Rabelais. Madrid: Alianza Editorial, 2005.

BARRANCOS, Dora. Mujeres, entre la casa y la plaza. Buenos Aires: Sudamericana, 2008.

BHABHA, Homi. El lugar de la cultura. Buenos Aires: Manantial, 2002.

BINAYÁN CARMONA, Narciso. Pasado y permanencia de la negritud. Todo es Historia. Buenos Aires, no 162, p. 66-72, noviembre 1980.

BOURDIEU, Pierre; WACQUANT, Loïc. Sur les ruses de la raison impérialiste. Actes de la recherche en sciences sociales. Vol. 121-122, p. 109-118, mars, 1998. Disponible à: https://www.persee.fr/doc/ arss_0335-5322_1998_num_121_1_3250 Accès: 15 dec. 2019.

CHAMOSA, Oscar. To Honor the Ashes of their Forebears: The Rise and Crisis of African Nations in the Post-Independence State of Buenos Aires, 1820-1860. The Americas. A Quarterly Review of InterAmerican Cultural History. V. 59, no 3, p. 347378, 2003. Disponible en: https://www.jstor.org/ stable/1008502?seq=1 Accès au: 15 nov. 2019. 
CHAVES, Mariana. Jóvenes, territorios y complicidades. Una antropología de la juventud urbana. Buenos Aires: Espacio Editorial, 2010.

CIRIO, Norberto Pablo. Tinta negra en el gris de ayer: los afroporteños a través de sus periódicos entre 1873 y 1882. Buenos Aires: Teseo, 2009.

CIRIO, Norberto Pablo. ¿Cómo suena la música afroporteña hoy? Hacia una genealogía del patrimonio musical negro de Buenos Aires. Revista del Instituto de Investigación Musicológica "Carlos Vega". Buenos Aires, Año 21, № 21, p. 84-120, 2007. Disponible en: http://bibliotecadigital.uca.edu.ar/repositorio/revistas/como-suena-musica-afroportena. pdf [consultado el 27 dic. 2019].

CONCOLORCOVO. El lazarillo de ciegos caminantes desde Buenos Aires hasta Lima, 1773. Buenos Aires: Biblioteca de la Junta de Historia y Numismática Americana, 1942.

ESCOBAR, Arturo. Culture, economics, and politics in Latin American social movements theory and research. In ESCOBAR, Arturo; ÁLVAREZ, Sonia. The making of social movements in Latin American: Identity, strategy, and democracy. Boudler: Westview press, 1992. p. 62-85.

ESTRADA, José Manuel. El tambor, en Almanaque Agrícola, Industrial y Comercial de Buenos Aires. Buenos Aires: P. Morta Editor, 1863.

FERREIRA, Luis. El movimiento negro en Uruguay (1988-1998): una versión posible. Montevideo: Ediciones Étnicas Mundo Afro, 2003.

FERREIRA, Luis. Dimensiones afro-céntricas en la cultura performática uruguaya. In: GOLDMAN, Gustavo (Comp.). Cultura y sociedad afro-rioplatense. Uruguay: Perro Andaluz, 2008. p. 91-123.

FRIGERIO, Alejandro; LAMBORGHINI, Eva. El candombe (uruguayo) en Buenos Aires: (Proponiendo) Nuevos imaginarios urbanos en la ciudad blanca. Cuadernos de Antropología Social. Buenos Aires, № 30, p. 93-118, 2009. Disponible à: http://revistascientificas.filo.uba.ar/index.php/CAS/article/ view/2778 Accès au: 15 nov. 2019.

FRIGERIO, Alejandro; LAMBORGHINI, Eva. Los afroar- gentinos: formas de comunalización, creación de identidades colectivas y resistencias culturales y políticas. In CATTERBER, Gabriela; MESCADO, Ruben (Comp.). Aportes para el desarrollo humano en Argentina. Afrodescendientes y africanos en Argentina. Buenos Aires: PNUD, 2011. p. 1-51.

GELER, Lea. ¿Quién no ha sido negro en su vida? Performances de negritud en el carnaval porteño de fin de siglo (XIX-XX). In GARCíA JORDÁN, Pilar (edit.). El Estado en América Latina. Recursos e imaginarios, siglos XIX-XXI. Barcelona: Publicacions de la Universitat de Barcelona, 2011. p. 183-211.

GHIDOLI, María de Lourdes. Acerca de la transparencia de Candombe Federal de Martín Boneo. In: Jornadas de Estudios Afrolatinoamericanos del GEALA, Instituto Ravignani, Facultad de Filosofía y Letras, Universidad de Buenos Aires, Anais... Buenos Aires, Mimeo, 2010. p. 1-13.

HALL, Stuart. Sin garantías. Trayectorias y problemáticas en estudios culturales. Quito: Envión Editores, 2010.

HALL, Stuart. Introducción: ¿quién necesita «identidad»? In. STUART, H. \& DU GAY, P. Cuestiones de identidad cultural. Buenos Aires: Amorrortu, 2003. p. 13-39.

HALL, Stuart. Notas sobre la deconstrucción de lo popular. In SAMUEL, Ralph. Historia popular y teoria socialista. Barcelona: Grijalbo, 1984. p. 1-11.

HELLEBRANDOVÁ, Klára. El proceso de etno-racialización y resistencia en la era multicultural: Ser negro en Bogotá. Universitas humanística. Bogotá, no 77, p. 145-168, enero-junio, 2014. Disponible à: https://revistas.javeriana.edu.co/index.php/univhumanistica/ article/view/5934 Accès au: 15. nov. 2019.

INGENIEROS, José. Sociología argentina. Madrid: Biblioteca Científico-filosófico, 1913.

KORDÓN, Bernardo. La raza negra en el Río de la Plata. Todo es Historia. Buenos Aires, suplemento no 7, p. 1-7, septiembre, 1968.

La Broma, 9 de marzo de 1882.

La Juventud, 10 de enero de 1879.

LAO-MONTES, Agustín. Decolonial moves: Trans-lo- 
cating African diaspora spaces. Cultural Studies, Harvard, Vol. 21, (2-3), p. 309-338, 2007. Disponible à: https://www.tandfonline.com/doi/ abs/10.1080/09502380601164361?src=recsys\&journalCode=rcus20 Accès au: 15 nov. 2019.

LAVOU ZOUNGBO, Victorien. Bartolomé de las Casas face à l'esclavage des Noir-e-s en Amérique/Caraỉbes. L'abération du Onzième Remède (1516). Perpignan: Presses Universitaires de Perpignan, 2011.

LÓPEZ, Laura. Identidades en juego alrededor del candombe. Revista de Investigaciones Folclóricas. Buenos Aires, no14, p. 91-96, 1999. Disponible à: https: / / pt.scribd.com/document/203113312/Identidades-en-Juego-Alrededor-Del-Candombe-laura-Lopez Accès au: 15 nov. 2019.

LÓPEZ, Vicente Fidel. Manual de la Historia Argentina dedicado a los profesores y maestros que la enseñan. Buenos Aires: C. Casavalle Editor, 1896.

NGOU-MVE, Nicolas. El origen bantú del kilombo iberoamericano siglos XVI y XVII. In: Kilombo. $N^{\circ}$ 1, Libreville: Centre d'Etudes et de Recherches Afro-Ibéro-Américaines, 2001. p. 61-77.

ORTIZ ODERIGO, Néstor. Las naciones africanas. Todo es Historia. Buenos Aires, no 162, p. 28-34, 1980.

PISTONE, Catalina. La esclavatura negra en Santa

Fe. Santa Fe: Junta Provincial de Estudios Históricos de Santa Fe, 1996.

PRATT, Mary Louise. Ojos imperiales. Literatura de viajes y transculturación. México: Fondo de Cultura Económica, 2010.

QUESADA, Vicente. Memorias de un viejo. Buenos Aires: Fondo nacional de las Artes, 1998.

RAHIER, Jean. Blackness, the racial/spatial order, migrations and Miss Ecuador 1995-96. American Anthropologist, 100(2), p. 421-430, 1998. Disponible à: https://www.jstor.org/stable/683121?seq=1 Accès au: 15 nov. 2019.
RAMOS MEJÍA, José María. Rosas y su tiempo. Tomo I. Buenos Aires: Félix La jouane y Cía. Editores, 1907.

REGUILLO, Rossana. Culturas juveniles. Formas Políticas del desencanto. Buenos Aires: Siglo XXI Editores, 2012.

RODRIGUEZ MOLAS, Ricardo. Negros libres rioplatenses. Revista de Humanidades, Buenos Aires, № 1, p. 99-126, septiembre, 1961.

SARMIENTO, Domingo Faustino. Conflictos y armonías de las razas en América. Buenos Aires: Editorial La Cultura, 1900.

SCHÁVELZON, Daniel. Buenos Aires Negra. Arqueología histórica de una ciudad silenciada. Buenos Aires: Emecé, 2003.

TAYLOR, Charles. El Multiculturalismo y la política de reconocimiento. México: FCE, 1993.

VÁZQUEZ, Cecilia. Arte y protesta: notas sobre prácticas estéticas de oposición. En ALABARCES, Pablo y RODRÍGUEZ, María Cecilia (comps.). Resistencias y mediaciones. Estudios sobre cultura popular. Buenos Aires: Paidós, 2008, pp. 165-188.

VILLANUEVA, Estanislao. El candombe nació en África y se hizo rioplatense. Todo es Historia, Buenos Aires, № 162, p. 44-58, 1980.

WILLIAMS, Raymond. Keywords. London: Fontana, 1988.

YÚDICE, George. El recurso de la cultura: usos de la cultura en la era global. Barcelona: Gedisa, 2002.

\section{Fuentes}

MATA LINARES, Benito. Colección de Reales Cédulas. Madrid: Academia de la Historia, Tomo II, Legajo 9/1657: F. 179-185 / F. 192-198.

Recebido em: 30.11.2019 Revisado em: 29.04.2020 Aprovado em: 12.05 .2020

Jean-Arsène Yao est Maître de conférences-Cames. Coordonnateur du Grelat (www.grelat-ufhb.org). Université Félix Houphouët-Boigny (Côte d'Ivoire). Ses axes de recherche son la construction intellectuelle de l'identité argentine et l'occultation de la présence-histoire des Noir-e-s; l'ethnohistoire des populations Afro-descendantes de l'Amérique Latine; l'étude des lieux et itinéraires de mémoire africains et afro-descendants en Amérique Latine. E-mail: jean.yao@univ-fhb.edu.ci 